\title{
ON LEADING SPIRAL ARMS IN CLOSE PAIRS OF GALAXIES
}

A. M. Fridman

Astronomical Council of the USSR Academy of Sciences

48, Pyatnitskaya Stree, 109017

MOSCOW, USSR

ABSTRACT. It is explained why one can observe a leading spiral pattern in close pairs of galaxies where the direction of the orbital momentum of the satellite is opposite to the direction of the spin of the spiral galaxy.

\section{INTRODUCTION}

One can hardly find any other problem in galaxy physics which has been discussed so long and experienced so frequently changing opinions, than the problem of the direction of winding of galactic spiral arms. According to the initial point of view followed from Lindblad theory (1), all spiral galaxies must have leading spiral arms. The observations however have not confirmed this conclusion (2). Though the interpretation was critisized in (3), following (4) scientists give preference to trailing spirals almost unanimously. But the currently prevailing point of view that all spirals are trailing seems too categorical. There are exceptions to this rule (5), and the analysis of their reasons is the aim of the present note. 
WHERE MAY THE LEADING GALACTIC SPIRAL ARMS BE OBSERVED?

Examining 190 spiral galaxies, Pasha has found that only three of them show leading arms (5). All the three have a close massive companion. One of them, NGC 5426, was studied more carefully; its spin is opposite to the orbital momentum. According to Werner $(6), 698$ of the paired galaxies show the same property.

\section{BASIC HYPOTHESIS}

Let us designate as leading those spirals which are rotating with their "tip" forward. Since the question is about the rotation of the spiral "tips," a peripheral region of the galactic disk is meant here. To identify a spiral as leading, it is enough for the largest part of the disk (including periphery) to be rotating in the same direction to which the spiral arms' tips are oriented. Let us now take a spiral to be trailing in the whole of the galactic disk except its periphery. Under the influence of the tidal wave from the galaxy's satellite, the main part of the disk (including periphery) is rotating opposite with respect to the rotation of the center part of the disk (Figure 1). Then determining the type of spiral with respect to the direction of rotation of the main part of the disk, we can make a conclusion about the leading form of the spiral pattern. 


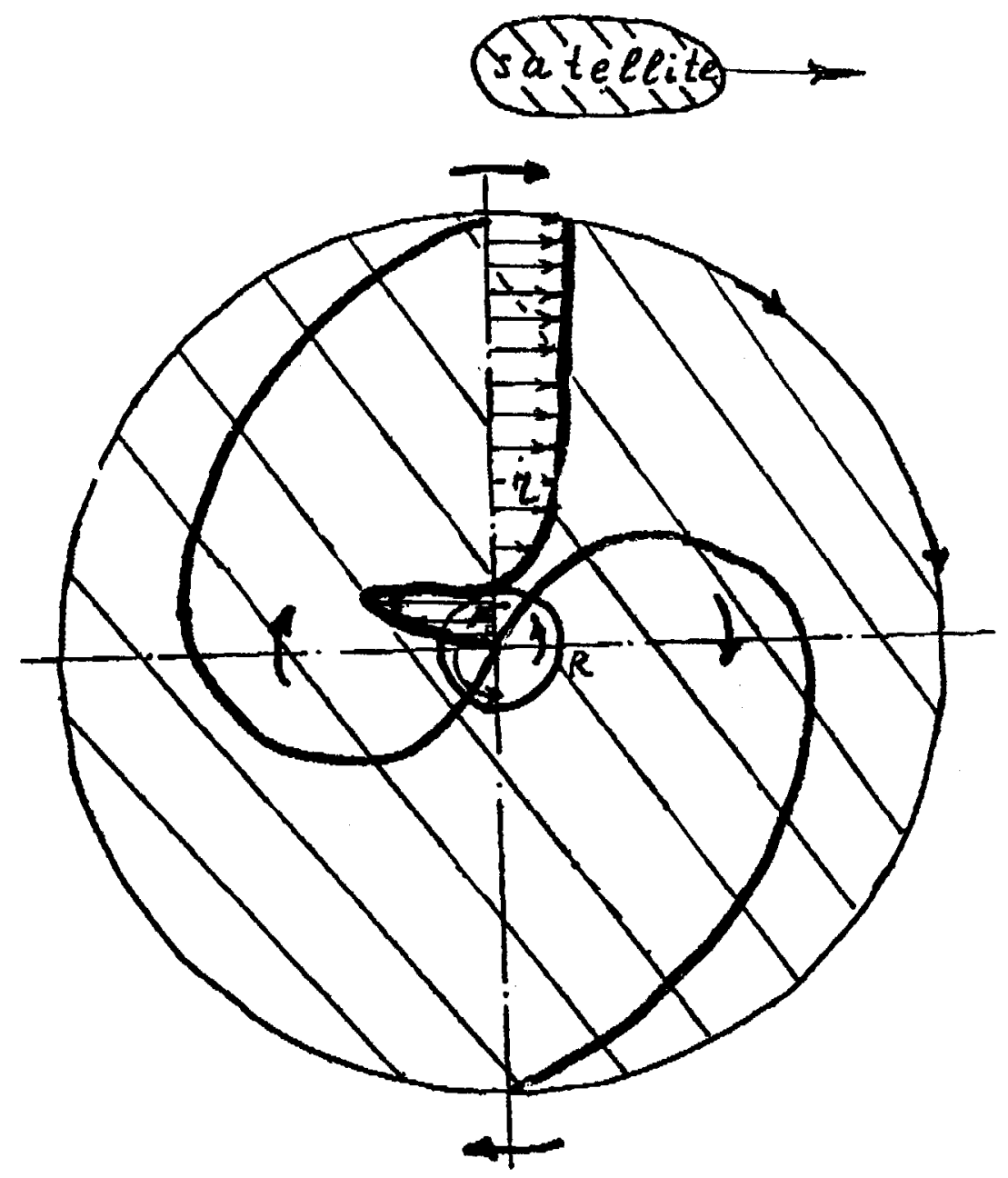

Figure 1. The center part of the spiral galaxy seen face-on rotating counter-clockwise; the main part of the disk (including the peripheral region) is rotating clockwise (as the satellite). Arrows show a vector field of linear velocities $\vec{V}_{O \phi}$ of the galactic disk and the satellite. On the radius $r$ - $R$ hodograph of motion has a "kink" which is typical of Vk-galaxies $(9,10)$. It can be seen in Figure 1 that the same galactic spiral is trailing in the center part of the disk's region, but in the main part of the disk (dotted) the spiral is leading. 
CONCLUSIONS OF THE THEORY

Last years' 6-meter telescope observations $(7,8)$ to determine the profiles of rotation velocities of the gas disk in spiral galaxies have shown that so-called VK-galaxies (with velocity kinks on their rotational velocity profiles $(9,10)$ ) "to be a rule rather than an exception" (8). So let us assume all the three spiral galaxies identified as leading in (5) to belong to VK-type. Then according to (10), the density waves are generated in the gas disk in the form of spiral arms characterized by the perturbed surface density $\sigma$ as the following function $\tau$ and $\phi$ :

$$
\sigma(\tau \phi) \sim \tau^{-1 / 2} \exp \left\{-\frac{\Omega^{2}{ }_{1} \mathrm{R} \tau}{\mathrm{Cg}_{1} \mathrm{Cg}_{2}} \cdot \frac{\left(1-\mathrm{Q}_{\mathrm{q}} 2\right)}{1+\mathrm{Q} \mu}+\operatorname{im}\left[\phi+\frac{\Omega_{1} \tau}{\mathrm{Cg}_{2}} \cdot \frac{(1-\mathrm{q})}{(1+Q \mu}\right]\right\}
$$

Here we have introduced the following designations:

$$
\begin{aligned}
& C_{g i}^{2} \equiv C_{s i}^{2}-\frac{2 \pi G \sigma_{o i}}{|K| R_{g}}, R_{g} \simeq \frac{1}{1+\frac{1}{2}|K| h_{g}}, C_{s i}^{2} \equiv \frac{d P_{o i}}{d \sigma_{o i}}, \\
& q \equiv \frac{\Omega_{2}}{\Omega_{1}}, Q \equiv \frac{\sigma_{1}}{\sigma_{2}}, \mu \equiv \frac{C_{2}}{C_{g}}, i=1,2 .
\end{aligned}
$$

$\mathrm{R}$ is the radius of kink velocity of the gas disk (Figure 1 ), $h$ is its typical thickness; parameters $q, Q^{-I}$ and $\mu$ characterize the 
relations of values of angular velocities, surface densities, and dispersions of gas velocities in exterior region $(r>R)$ to their values in interior regions $(r<R)$.

The augend in curly brackets (I) describes the decrease of the perturbed amplitude with radius, the addend $\sim i$ determines the form of the spiral pattern. If the latter is rewritten in the form of $k_{r} \tau+m_{\phi}=$ const, then

$$
K_{\tau}=m \frac{\Omega_{1}}{C g_{2}} \cdot \frac{(1-q)}{(p+Q \Gamma)}
$$

will correspond to the perturbation (1).

Let the interior region $(r<R)$ be rotating counter clockwise, i.e., $\Omega_{1}>\mathrm{Q}$. Then as follows from (2), if $q<1$ no matter in which direction the external region $(r>R)$ is rotating: $k_{r} / m>Q$, i.e., the spiral in the "wave meaning" (5) is trailing - it is rotating with its convex side forward. If the main part of the disk (including the periphery) is rotating in the opposite direction, the spiral will be rotating with its tips forward in this region, i.e., it will appear to be leading. 
CONCLUSION OF THE LABORATORY EXPERIMENT

In (10) it is proved that with the help of a simple redesignation the main dynamic equations of the self-gravitating gas disk transform in shallow water equations. It makes possible to use a model "Spiral" with rotating shallow water for modeling the generation process of the galaxies' spiral structure (1115). When the periphery was immovable $\left(\Omega_{2}=0, i . e ., q=0\right)$ density waves were trailing spirals (11-15). It is obvious from physics of generation process that if we rotate the periphery slowly (no matter in which direction), i.e., if we keep to the condition $|q|<<1$, the form of the spirals will change very little.

At the same time, with respect to the counter-rotating periphery (i.e., when $q<0$, but as before $|q|<<1$ ), the spiral waves will be oriented with their tips forward. The spiral in the "wave meaning" (5) will already be leading. Therefore, in all the region of the galactic disk, except for a small central part, the spiral will be oriented with its concave side in the direction of the rotation velocity vector. Without going into details, one can say that such a picture is observed in the laboratory experiment (13-15) (in detail see (15)). Thus, the region of the leading spiral (Figure 1) is wholly determined by the "trapped" region of the galactic disk by the tidal wave. Let us note in conclusion that it is simple to determine from (3) the angle of the spiral winding $\alpha \equiv \arctan (m / \overline{\bar{K}} \tau)$. 


$$
\alpha(\tau)=\arctan \frac{\mathrm{Cg}_{2}\left(1+Q_{\mu}\right)}{\Omega_{1} \tau(1-q)} .
$$

This formula can be experimentally checked substituting the parameters of the gas galactic disk by the parameters of the rotating shallow water and using (10). In particular, one can determine the relation tan a for leading and trailing spirals. If, for example, one manages all the parameters (except $q$ sign) in both cases to make constant, then the leading spiral in its relation to the trailing one must look more winding, because $(\tan \alpha) \operatorname{lead} /(\tan \alpha)_{\operatorname{tr}}=(1-|q|) /(1+|q|)$ and if $|a|$ $\ll 1$, this relation $* 1-2|q|$.

\section{ACKNOWLEDGMENTS}

I express my gratitude to F. A. Tsytsyn, D. A. Werner, and A. V. Zasov for useful discussions. 
REFERENCES

Afanasiev, V. L., et al. in The Tenth European Regional Astronomy Meeting of the IAU, Prague, Czechoslovakia, August 24-29, 1987.

Afanasiev, V. L., Burenkov, A. N., Zasov, A. V., Sil'chenko, O. K. 1988, Astrophys. J., 28, 243; 29, 155 .

Baev, P. V., Makov, Yu.N., Fridman, A. M. 1987, Pis'ma v Astron. $\underline{\text { Zh. }}, 13,964$.

de Vaucouleurs, G. 1958, Astrophys. J., 127, 487.

Fridman, A. M. 1989, in Dynamics of Astrohysical Disks, edited by J. Sellwood, Cambridge University Press, Cambridge, p. 185 .

Fridman, A. M., Morozov, A. G., Nezlin, M. V., Snezhkin, E. N. 1985, Physics Letters, 109A, 228.

Fridman, A. M., Horozov, A. G., Nezlin, M. V., Pasha I. I., Polyachenko, V. L., Pylov, A.Yu, Snexhkin, E. N., Torkashin, Yu.N., Trubnikov, A. S. 1987, In Observational Evidence of Activity of Galaxies, eds. Khachikian et al., by IAU, pp. 147157.

Hubble, E. 1943, Astrophys. J., 97, 112. 
Lindblad, B. 1940, Astrophys. J., 92, 1.

Lindblad, D., and Brahde R. 1945, Astrophys. J., 104, 211.

Morozov, A. G., Nezlin, M. V., Snezhkin, E. N., Fridman, A. M. 1984, Pis'ma ZHETF, 39, 504; Uspekhy Fiz. Nauk, 1985, 145, 161 .

Nezlin, M. V., Rylov, A.Yu., Snezhkin, E. N., Trubnikov, A. S. 1987, ZHETF, 92, 8.

Pasha, I. I. 1985, Pisma V Astron. Zh., 11, 3.

Werner, D. A. 1956, Astron. Tsirk., 1466, 5.

Zasov, A. V., Sil'chenko, O. K. 1987, Pis'ma V Astron. Zh, 13, 455 . 